\title{
The nature of a primary jet within a circumbinary disc outflow in a young stellar system
}

\author{
Chris Lynch ${ }^{1 \star} \&$ Michael D. Smith ${ }^{1} \dagger$ \\ ${ }^{1}$ Centre for Astrophysics \& Planetary Science, The University of Kent, Canterbury, Kent CT2 7NH, U.K.
}

Accepted ..... Received ..... ; in original form .....

\begin{abstract}
Most stars form in binaries, and both stars may grow by accreting material from a circumbinary disc onto their own discs. We suspect that in many cases a wide molecular wind will envelope a collimated atomic jet emanating from close to an orbiting young star. This so-called Circumbinary Scenario is explored here in order to find common identifiable properties. The dynamical set up is studied with three dimensional simulations with chemistry and cooling included. We extract the properties on scales of order $100 \mathrm{AU}$ and compare to the Co-Orbital Scenario in which the wind and jet sources are in orbit. We find that the rapid orbital motion generates a wide ionised sheath around the jet core with a large opening angle at the base. This is independent of the presence of the surrounding molecular outflow. However, the atomic jet is recollimated beyond $\sim 55 \mathrm{AU}$ when the molecular outflow restricts the motion of the ambient medium which, in turn, confines the jet. These physical properties are related to the optical $\mathrm{H} \alpha$ imaging, providing a means of distinguishing between models. The high excitation sheath and recollimation region can be explored on these scales through the next generation of instruments. However, in general, the amount and location of the ionised material, whether in the knots or the sheath, will depend on several parameters including the orbital period, axis alignment and pulse amplitude.
\end{abstract}

Key words: hydrodynamics - ISM: jets and outflows - stars: formation - stars: pre-mainsequence

\section{INTRODUCTION}

Spectacular outflows are often observed as a star forms. They occur during the phase when we expect inflows to be dominant (e.g. Smith 2004). Many protostars have been discovered through their infrared jets while the driving internal activity is highly obscured (McCaughrean et al. 1994; Zinnecker et al. 1998). A widely accepted resolution is that the inflow is held up by centrifugal forces in a rotating disc. The outflow then functions as the channel through which the angular momentum of the disc is gradually extricated. In support, there are several plausible mechanisms which may operate during accretion on to a single star to produce an outflow. These lead to extended disc winds, magnetic tower flows and magnetospheric X-winds (Blandford \& Payne 1982; Shibata \& Uchida 1985).

We should not neglect that most stars are not formed alone. For each binary, we might expect up to three outflow systems associated with three distinct discs with the large circumbinary disc feeding both a circumprimary disc and a circumsecondary disc. It follows that multiple outflow systems should be prevalent. Examples include the jets from both components of the XZTau binary

\footnotetext{
* E-mail: captainlockheed@btopenworld.com

† E-mail: m.d.smith@kent.ac.uk,
}

(Krist et al. 2008). Multiple outflow systems have also been recently confirmed for L 1551İRS5 (de Miera et al. 2019). Moreover, even the older T Tauri binary V582 Mon has been found to be surrounded by a circumbinary ring while driving a molecular outflow (Aronow et al. 2018). Yet, the observational evidence for multiple outflows remains very scattered. This leads us to ask how we should recognise multiple outflows. The approach to the problem taken here is through numerical simulations.

We aim to identify the formation processes by analysing how the outflows release their kinetic energy during interactions. The concept of orbiting outflows was first studied numerically by Murphy et al. (2008) where two adjacent atomic jets interact. Both jets were narrow and had a short dynamical flow time so that the long orbital time had little influence. This was remedied in Paper 1 (Lynch et al. 2019) where the Co-orbital Scenario was fully explored. In that case, as illustrated in Fig. 1, it is the two orbiting young stars which are the effective origins. The molecular outflow originates from from the secondary binary partner, as discussed by Tambovtseva \& Grinin (2008). The interaction is through direct impact as a wide molecular wind clashes and spirals around the atomic jet. Regions of warm ionised gas are generated off-axis and this yield strong $\mathrm{H} \alpha$ emission patches.

Here, we study the alternative Circumbinary Scenario in which the inner jet performs a short-period orbit, remaining within 
a wide circumbinary outflow cone. This scenario was suggested by Estalella et al. (2012) as an interpretation for the HH 30 outflows in which all the above components have been identified.

The production of a collimated fast jet enclosed by a wide low-speed outflow was uncovered in by Machida et al. (2008) in simulations. These outflows arise during the very early protostellar stage associated with the outer adiabatic core and the second protostellar core. This was confirmed in three-dimensional magnetohydrodynamics simulation by Tomida et al. (2013), Bate et al. (2014), Machida (2014) and Machida \& Basu (2019). The jet was found to propagate within the outflow, forming a nested structure in the velocity. The low-velocity outflow is in a nearly steady state, while the high-velocity jet appears intermittently due to a variety of inner disc instabilities. Although the jet carries the bulk of the kinetic energy, the wide outflow channels the mass and momentum. Here, we extend this work by studying the dual outflows and determining the physical properties which may lead to observable consequences.

In Paper 1 (Lynch et al. 2019), we summarised the interpretations and parameters which have been previously found to be consistent with both models as applied to HH 30 . We also detailed the physical, chemical and dynamical modifications to the ZEUS-MP code (Hayes et al. 2006), and listed the full set of simulations. Radiation energy, magnetic flux and gravitational forces are neglected. The full code includes a three-species chemistry model for hydrogen that evolves $\mathrm{H}_{2}, \mathrm{H}$ and $\mathrm{H}^{+}$, according to the model presented in Glover \& Mac Low (2007). The cooling of the gas includes that due to both atoms and molecules. The atomic cooling assumes a time-independent function and fine-structure cooling while molecular cooling includes vibrational and rotational contributions from $\mathrm{H}_{2}, \mathrm{CO}, \mathrm{OH}$ and $\mathrm{H}_{2} \mathrm{O}$. Dissociative cooling and dust cooling are also included.

The above model concepts are motivated by the HH 30 system which exhibits a well-collimated plume at visible wavelengths due to hot atomic and partially ionised hydrogen gas (Gardner et al. 2018), surrounded by a colder, dense, wide-angle molecular outflow (Hartigan \& Morse 2007). In the Circumbinary Scenario, the binary system is configured to be very close and the wiggle in the atomic jet is caused by tidally-induced precession. In this case, the molecular outflow is generated from the circumbinary disc, whose inner radius is small ( $3 \mathrm{AU}$ ) because of the tight binary orbit (0.75 AU).

It should also be remarked that the orbital and disc rotation axes are taken to be aligned in this work. This probably minimises the interaction; misalignment between the outflow axes is likely (Hirano \& Machida 2019) and merits further study. On the other hand, the remarkable collapse simulations described above have not quite reached the capability to yield binary protostellar cores, discs and sets of jets.

\section{METHOD}

Parameters for the circumbinary simulations were fixed by the following considerations. Kinematic and morphological modelling of the slow molecular outflow associated with HH 30 by Tambovtseva \& Grinin (2008) showed that the closest resemblance occurred when a very short-period binary orbit and a circumbinary disc source were taken. As a result, the jet wiggling is attributed to precession of the atomic jet source. We adopt a set of parameters based on the HH 30 outflow (defined as Type II in Table 4 of Paper 1). In summary, we take a tight binary with an orbital separation of $0.75 \mathrm{AU}$. In this Type II circumbinary model, estimates put the primary and secondary masses as $\sim 0.44 \odot$ and $\sim 0.1 \mathrm{M}_{\odot}$ put the total mass at $\sim 0.54 \mathrm{M}_{\odot}$. The orbital period is one year.

The speed and mass flow rate of the injected jet are 326 $\mathrm{km} \mathrm{s}^{-1}$ and $5.10 \times 10^{-9} \mathrm{M}_{\odot} \mathrm{yr}^{-1}$. The axis of this primary jet performs a motion in a cone as the driving disc is subject to precession with a period of 53 years with a half angle of just 0.025 radians $\left(1.43^{\circ}\right)$.

The speed and mass flow rate of the injected molecular wind are $11.7 \mathrm{~km} \mathrm{~s}^{-1}$ and $6.15 \times 10^{-8} \mathrm{M}_{\odot} \mathrm{yr}^{-1}$. This wind, from a disc in fixed Keplerian rotation, stems from inner and outer radii of $9-$ $21 \mathrm{AU}$.

The speed of the jet is variable on short and long timescales. The long period variability in the speed of ejection would probably lead to the knots of bright emission observed in the HH30 outflow on the scale of 0.1 parsec (Raga et al. 1990). The short-period variability ( $\sim$ months), which might be chaotic in nature, is triggered from variable accretion. The resulting compressions steepen into shock fronts that provide the collisional heating and ionisation of the jet material (Hartigan \& Morse 2007; Anglada et al. 2007). Furthermore, the HH 30 jet appears to be driven sideways on $t$ he parsec scale in the sky plane. It displays a curved West-facing 'C' shape that may be caused by systemic intrinsic velocity changes, or impinging outflows or winds from nearby objects (Estalella et al. 2012).

The numerical calculations were performed using MPI with ZEUS-MP employing 180 tiles on the SCIAMA supercomputer. The desire for high resolution in three dimensions despite the strong lateral interaction restricted the grid length to just over $100 \mathrm{AU}$. After much experimenting, we settled on the values provided in Table 1. In the $\mathrm{x}$-dimension along the axis, 20 thin slabs each of 8 zones in width are stacked. Each of these slabs consist of a $3 \times 3$ arrangement of square $115 \times 115$ zone tiles in the $y-z$ plane.

Despite being inspired by HH 30 , the consequences of the more general problem as sketched in the left panel of Fig. 1 is of interest. To this end, a broad range of simulations were first performed until a small number of the most relevant conditions could be selected for high resolution study. Table 2 summarises the characteristics of the circumbinary simulations designated by the prefix s067. Major long timescale ( $175 \mathrm{yrs})$ runs were performed to establish fully developed flow structures. The atomic jet model was also run without the molecular flow.

The jet diameter is eleven cells, corresponding to $1.1 \times{ }^{14} \mathrm{~cm}$, commensurate with the assumed distance from the driving star of order $10 \mathrm{AU}$. The initial temperature and ion fraction in the jet are fixed at arbitrary values with the pulses immediately raising them to appropriate vales. The circumbinary disc has an inner radius of $1.4 \times 10^{14} \mathrm{~cm}$, Mach number of 30 and a rotation speed of $6.5 \times 10^{-9}$ radians s$^{-1}$ which yields $9.1 \mathrm{~km} \mathrm{~s}^{-1}$. This creates a molecular wind with an opening angle of $\sim 67^{\circ}$.

We then performed six additional runs of shorter duration. These simulations; three with the velocity pulse time periods for the atomic jet varied and three with different values for the orbital eccentricity taken. The data retained for these simulations start from 65 years into the outflow evolution (the outflows having crossed the problem domain by this time and so display a fully developed flow) and traces the development for the ensuing 22 years.

The period of the pulsed velocity $T_{\text {vpulse }}$ corresponds to the period of the sinusoidal signal imposed on the velocity of injected material. In all the simulations presented in these studies, the Relative Amplitude parameter $A_{R}$ used is set to 0.2. This generates a 


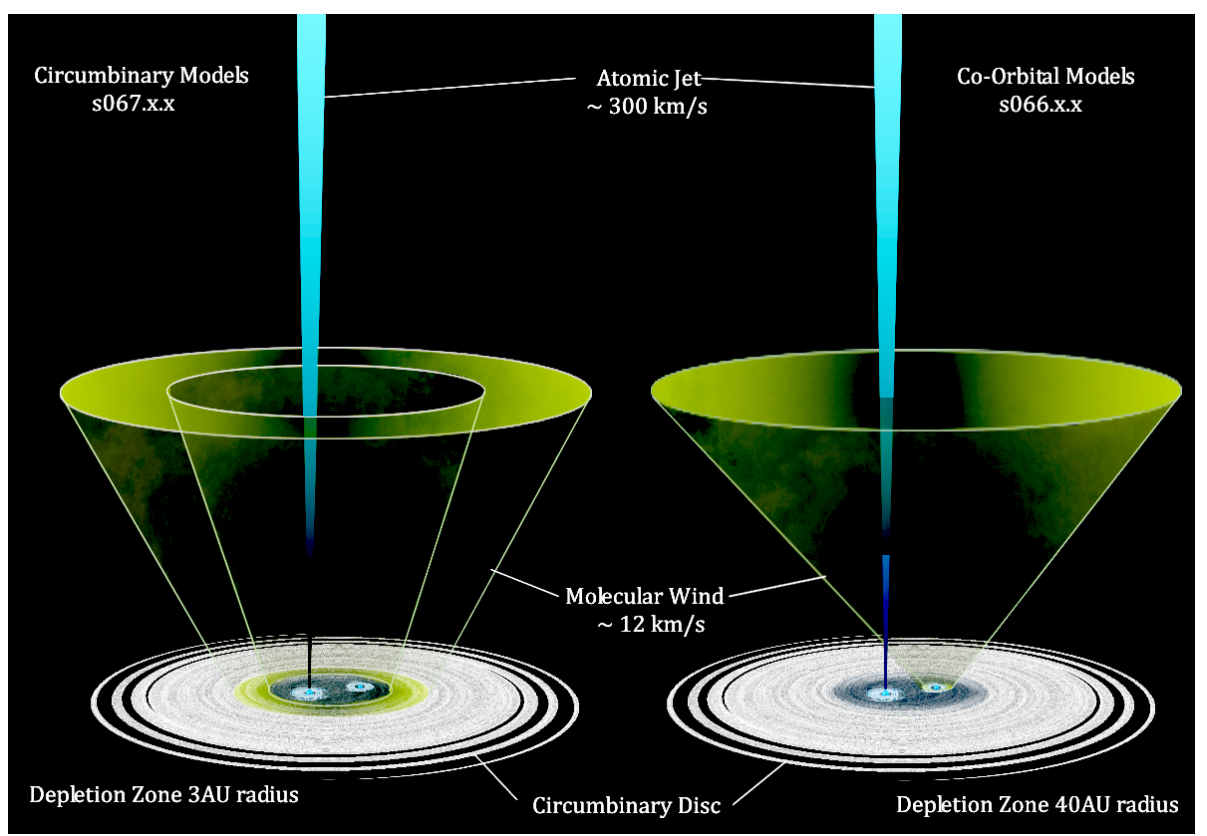

Figure 1. An illustration of the Circumbinary and Co-orbital Scenarios. A simulation was performed within each model series without the molecular flow as a control.

Table 1. Standard model geometry and tiling

\begin{tabular}{crrrrrrr}
\hline Coordinate & $\begin{array}{r}\text { Min } \\
(\mathrm{cm})\end{array}$ & $\begin{array}{r}\text { Max } \\
(\mathrm{cm})\end{array}$ & $\begin{array}{r}\text { Span } \\
(\mathrm{cm})\end{array}$ & $\begin{array}{r}\text { Grid } \\
\text { Zones }\end{array}$ & $\begin{array}{r}\text { Zone Size } \\
(\mathrm{cm})\end{array}$ & $\begin{array}{r}\text { MPI } \\
\text { Tiling }\end{array}$ & $\begin{array}{r}\text { Zones } \\
/ \text { Tile }\end{array}$ \\
\hline $\mathrm{x}$ & 0 & $1.600 \times 10^{15}$ & $1.600 \times 10^{15}$ & 160 & $1.0 \times 10^{13}$ & 20 & 8 \\
$\mathrm{y}$ & $-1.725 \times 10^{15}$ & $1.725 \times 10^{15}$ & $3.450 \times 10^{15}$ & 345 & $1.0 \times 10^{13}$ & 3 & 115 \\
$\mathrm{z}$ & $-1.725 \times 10^{15}$ & $1.725 \times 10^{15}$ & $3.450 \times 10^{15}$ & 345 & $1.0 \times 10^{13}$ & 3 & 115 \\
\hline
\end{tabular}

Table 2. Simulation Runs

\begin{tabular}{|c|c|c|c|c|c|c|}
\hline Simulations & $\begin{array}{c}\text { Outflows } \\
\text { Atomic }\end{array}$ & Molecular & $\begin{array}{l}\text { Vpulse } \\
\text { Period }\end{array}$ & $\begin{array}{c}\text { Orbital } \\
\epsilon\end{array}$ & $\mathrm{T}_{1}(\mathrm{yrs})$ & $\mathrm{T}_{2}(\mathrm{yrs})$ \\
\hline s067.3.2 & II & II & $5.256 \times 10^{6}$ & 0.00 & 0 & 175 \\
\hline s067.3.3 & II & - & $5.256 \times 10^{6}$ & 0.00 & 0 & 175 \\
\hline s067.4.3 & - & II & & - & 0 & 80 \\
\hline s067.4.3 & II & II & $1.051 \times 10^{7}$ & 0.00 & 65 & 87 \\
\hline s067.4.4 & II & II & $7.884 \times 10^{6}$ & 0.00 & 65 & 87 \\
\hline s067.4.5 & II & II & $1.314 \times 10^{7}$ & 0.00 & 65 & 87 \\
\hline s067.5.25 & II & II & $5.256 \times 10^{6}$ & 0.25 & 65 & 87 \\
\hline $\mathrm{s} 067.5 .50$ & II & II & $5.256 \times 10^{6}$ & 0.50 & 65 & 87 \\
\hline $\mathrm{s} 067.5 .75$ & II & II & $5.256 \times 10^{6}$ & 0.75 & 65 & 87 \\
\hline
\end{tabular}

velocity signal of the form

$$
V_{J}(t)=M \times C_{J} \times \frac{1+A_{R} \cos \omega_{v} t}{1+A_{R}},
$$

where $M$ is the jet Mach number, $C_{J}$ is the jet sound speed, and $\omega_{\mathrm{v}}=2 \pi / T_{\mathrm{vpulse}}$. The maximum of this signal is $M C_{J}$ and the minimum is $66 \%$ of this value. The Keplerian orbit of the jet inlet is derived from the orbital eccentricity parameter, $\epsilon$. The NewtonRaphson method is used in this calculation. The dumps of HDF data are saved at simulation time intervals of $2.125 \times 10^{6}$ seconds. This value is chosen to be less than half of $T_{\mathrm{vpulse}}$. This is the standard strategy in signal processing: in order to capture a sinusoidal signal the sampling frequency must be at least twice the highest frequency component of the signal. In addition, with the choice of 
$T_{S} \approx 2.5 T_{\text {vpulse }}$, we avoid 'strobe' effects which can spoil animations. We may also proceed to determine proper motions of knots and other structures.

\section{RESULTS}

\subsection{A single atomic jet on a tight orbit}

The simulation designated as s067.3.3 models a scenario in which only the fast-moving, atomic jet is launched by the more massive binary partner in the two-star system. The molecular outflow is absent. The immediately striking feature evident in the cross-sections of Figure 2 is the plume of partly ionised gas filling the cocoon around the jet. Shown in green on this figure, the ion fraction is about ten times higher than in the co-orbital equivalent.

This broad ionised sheath has its explanation in the tight fast orbit which has the effect of spraying the jet over a wide area rather than allowing material to penetrate unhindered through an already evacuated narrow channel. The rapidly orbiting jet source spreads the ejected gas over a larger area. Remarkably, this leads to a wide conical distribution for the ionised sheath in the initial few AU, as shown in Panel (d), which could be mistakenly identified with the jet before collimation has been achieved.

The high excitation sheath was not found in Paper 1. The difference between the present scenario and the co-orbital model is the orbital period. This model has a binary orbit separation of just $0.75 \mathrm{AU}$, while the co-orbital jet had an $18 \mathrm{AU}$ separation. Essentially, this jet remains in place and 'wiggles' furiously about the barycentre with a Keplerian orbital velocity of $\sim 26 \mathrm{~km} \mathrm{~s}^{-1}$, while the long-period co-orbital jet moves gradually through the ambient medium at $\sim 5 \mathrm{~km} \mathrm{~s}^{-1}$.

A series of high-density knots rapidly develops within the jet from the sinusoidal pulsed signal that begins purely as a velocity perturbation, as displayed also in Panels (d) and (h) of Fig. 3 at the later fully-developed time. There are eleven distinct density knots spanning the problem domain. The ionisation fraction is low within the jet knots but there is a moderate increase towards the jet-cocoon interface.

Pressure and temperature distributions are also displayed in the lower panels of Fig. 3 for the the simulation time of 175 years. It is evident from these panels that the low-density cocoon surrounding the jet column, as well as the low-density regions within the jet, are populated by hot, partially ionised material. The interface with the ambient medium develops transverse structure probably related to the Kelvin-Helmholtz instability. The velocity along the jet direction is suppressed within the sheath but the speed remains in in the range $100-300 \mathrm{~km} \mathrm{~s}^{-1}$ across the jet.

\subsection{Dual Atomic-Molecular Outflow (simulation s067.3.2)}

The molecular outflow, emerging from the circumbinary disc, is now introduced along with the above atomic jet. The set of crosssections of density is displayed in Figure 4 for the simulation time of 87.5 years. All the figure share a common colour scaling of variables which permits a direct comparison.

The morphology of the molecular outflow strongly supports the ad hoc and ballistic particle models calculated by Pety et al. (2006) and Tambovtseva \& Grinin (2008). The lateral expansion is driven mainly by the Keplerian rotational velocity profile rather than the pressure differential, although the outflow is $1.5 \times$ overpressured with respect to the ambient medium. This an important

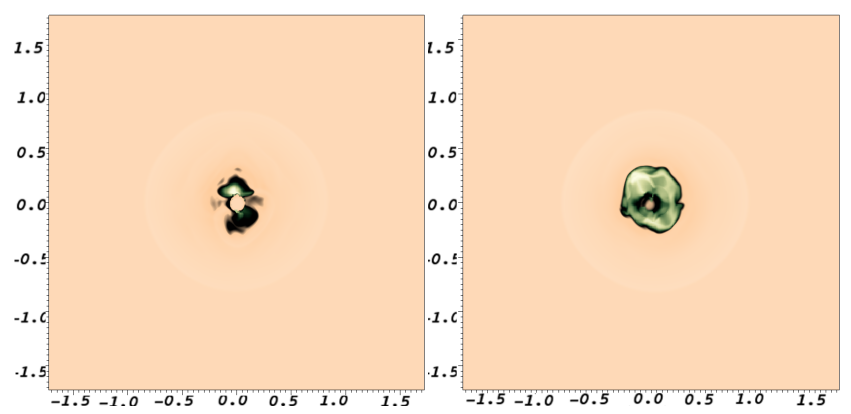

(a) $z-y$ plane, $x=0 \mathrm{~cm}$

(b) $z-y$ plane, $x=7.5 \times 10^{14} \mathrm{~cm}$

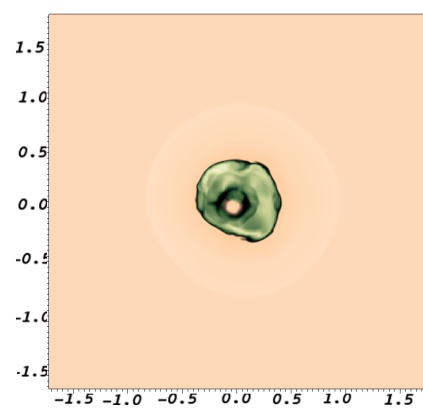

(c) $z-y$ plane, $x=1.5 \times 10^{15} \mathrm{~cm}$

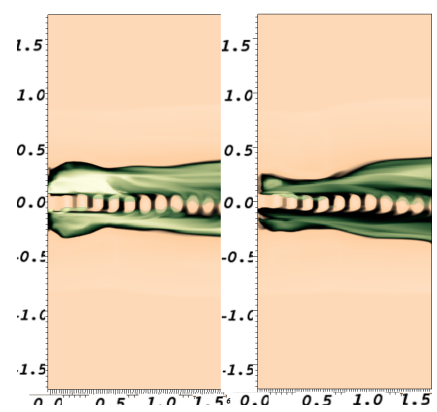

(d) $x-y$ plane, $\mathrm{z}=0 ; x-z$ plane, $\mathrm{y}$ $=0$
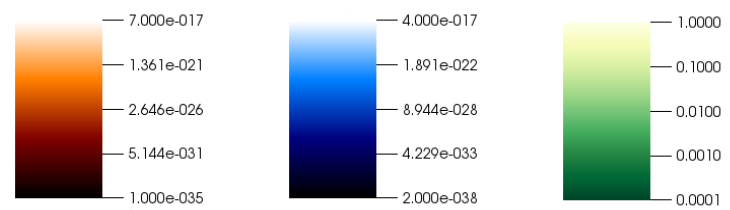

(e) Density $\left(\mathrm{g} \mathrm{cm}^{-3}\right)$ (f) $\mathrm{H}_{2}$ density $\left(\mathrm{g} \mathrm{cm}^{-3}\right) \quad$ (g) Fractional ion.

Figure 2. Single atomic jet. Cross-sections under conditions of a $0.75 \mathrm{AU}$ binary, at simulation time 87.5 years. The axis scales are in units of $10^{15} \mathrm{~cm}$. The ambient medium is atomic with traces of molecular hydrogen formed during the simulation. The underlying density plot is fully opaque. For clarity, $\mathrm{H}_{2}$ (blue) and ionisation fraction (green) overplots are at ramped opacity: $100 \%$ opacity at maximum value, fully transparent at minimum value.

contrast with the Co-orbital dual-outflow model. We see the expanding remnant of the bow shock in the ambient medium as before but this is now dominated by the broader bow shock of the molecular outflow.

The features of the inner part of the central pulsed atomic jet column are virtually identical to those already identified in the atomic-only case. The ionised sheath, however, is compressed from the base by the proximity to the high-pressure disc outflow. This has squeezed the sheath which then remains relatively narrow, as shown in Panel (d) of Figure 4.

In Panel (b) we see that a low density cavity has been carved out from the ambient medium enclosed by the conical molecular flow. This kind of evacuated region does not have an equivalent in the atomic-only simulation. The ambient medium is trapped, prevented from flowing back into the cavity by waves of hot, light, partially ionised material expanding out from the atomic jet periphery and impacting against the boundary of the denser material. As 

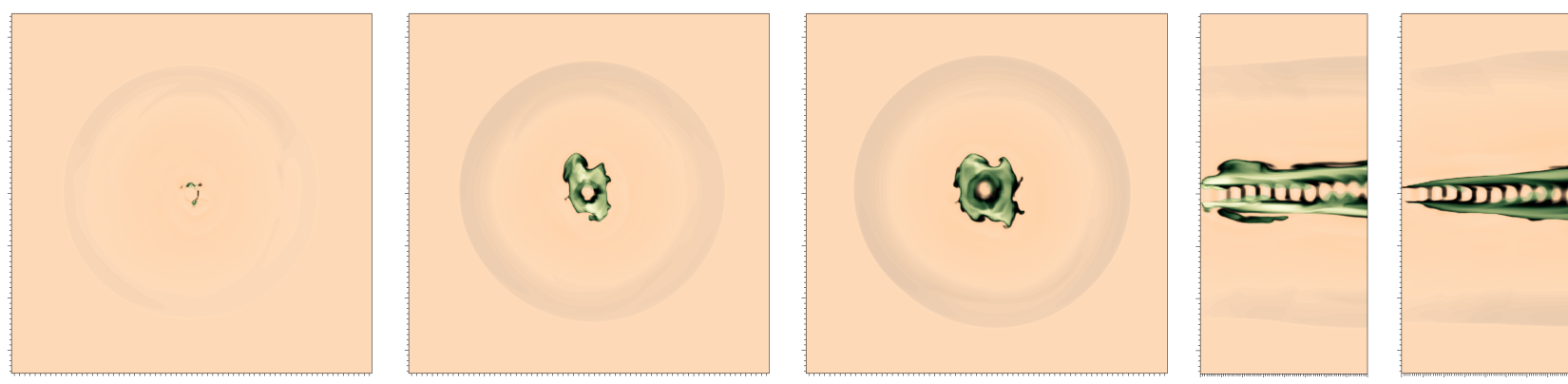

(a) density and composition at $\mathrm{x}=0 \mathrm{~cm}$ (b) density and composition, 50AU

(c) density and composition, 100AU (d) density and composition, xz plane

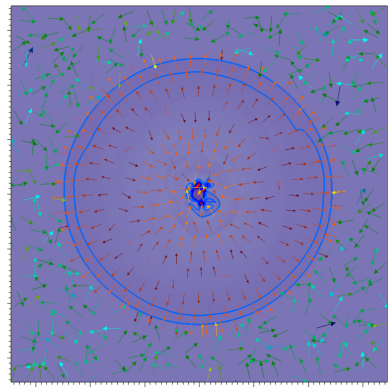

(e) $\mathrm{p}, \mathrm{T}$ and $\mathrm{v}$ at $\mathrm{x}=0 \mathrm{~cm}$

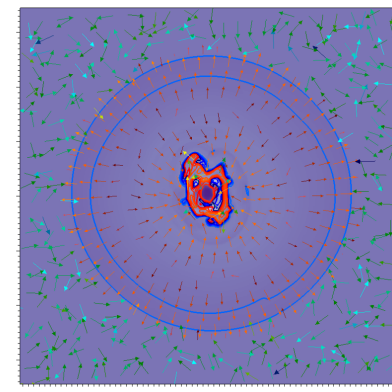

(f) $\mathrm{p}, \mathrm{T}$ and $\mathrm{v}$ at $\mathrm{x}=50 \mathrm{AU}$

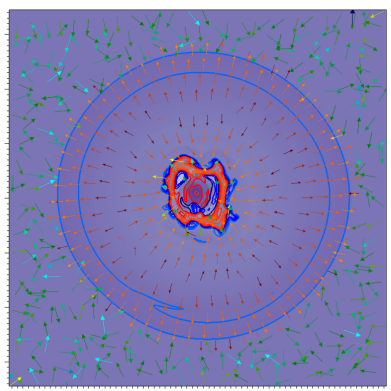

(g) p, T and $\mathrm{v}$ at $\mathrm{x}=100 \mathrm{AU}$

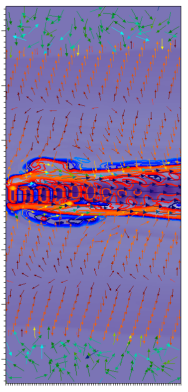

(h) p, T and $v$ in $x y$ and $x z$ planes

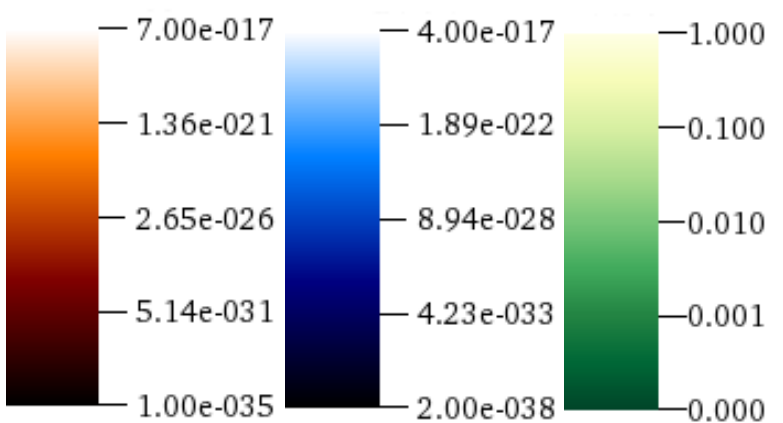

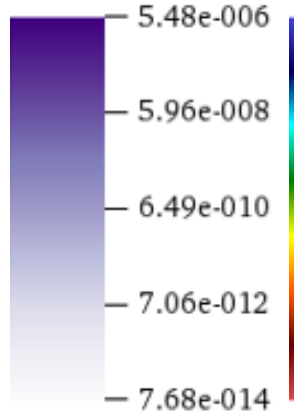

(1) pressure

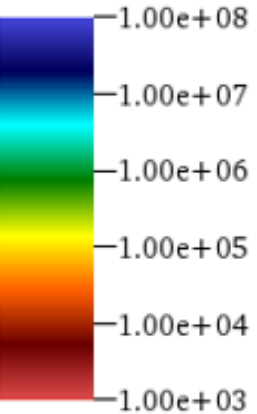

(m) velocity vectors

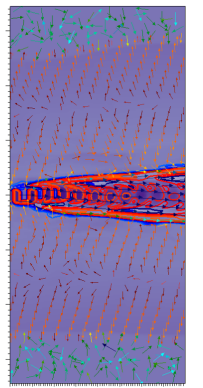

$-230000$

$-75000$

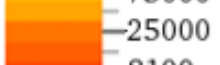

- 8100

$-2600$

$-870$

$-280$

$-90$

90

-30
-10
(i) density, $\mathrm{H}$
(j) density, $\mathrm{H}_{2}$
(k) ion fraction

Figure 3. Cross-sections of physical parameters for the single atomic jet at the later simulation time of 175 years. Axis scales are in units of $10^{15} \mathrm{~cm}$. Ambient medium is atomic hydrogen with trace molecular hydrogen formed during the simulation. Underlying density plot is fully opaque.

the simulation progresses (see Fig. 5) the cavity is slowly inflated as the ambient material is eroded.

\subsection{Temperature and Excitation}

The temperature profiles across the lateral span of the domain are shown in Figure 6 at three distances along the barycentric (x) axis. Note the logarithmic temperature scale. The outer 'shoulders' correspond to the bow shock from the molecular outflow, propagating outwards at the medium sound speed.

Deep troughs in the temperature profile correspond to the cold molecular material from the circumbinary disc while the central high plateau shows the presence of the jet. Note that the region immediately surrounding the jet is hotter than the jet itself. The very high temperatures developed in the periphery of the jet increase with distance from the jet inlet boundary (see legend), while the temperature in the jet column is decreasing. Clearly, the warm atomic jet maintains a constant width of $\sim 20 \mathrm{AU}$ while the narrow shear layer in which kinetic energy has been dissipated separates the atomic jet from the ambient cavity.

In order to understand the ionisation process in this model, it is instructive to examine the velocity behaviour of material in the jet as it orbits tightly about the barycentre. Figure 7 compares the centre-line velocity signal down the middle of the jet with the velocity variation on the jet periphery.

The velocity signal at the jet inlet is $2.717 \pm 0.543 \times 10^{7} \mathrm{~cm} / \mathrm{s}$ varying sinusoidally. The centre-line velocity eventually settles at a mean value of $2.746 \pm 0.014 \times 10^{7} \mathrm{~cm} / \mathrm{s}$ at the outgoing boundary; though there is some suggestion in the signal that the velocity amplitude might be re-intensifying in line with the findings of Smith et al. (1997). It is evident that the velocity difference at the jet periphery is greater than within the jet column itself with an initial steep fall of $54 \%$ from the value at the inlet boundary over the first 


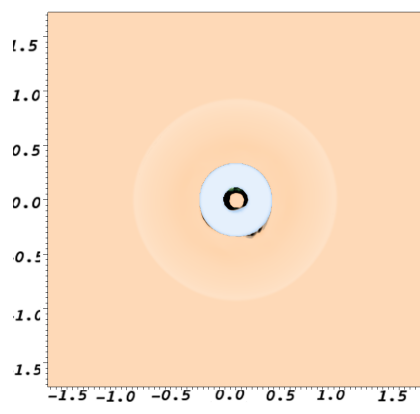

(a) z-y plane, $x=0 \mathrm{~cm}$

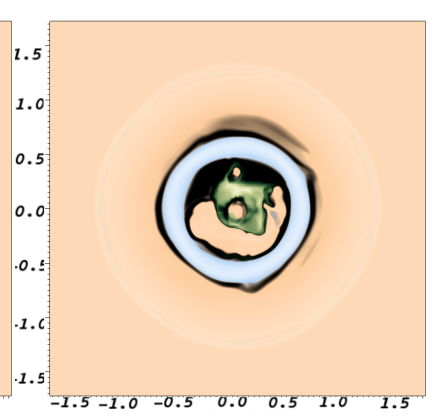

(b) z-y plane, $x=7.5 \times 10^{14} \mathrm{~cm}$

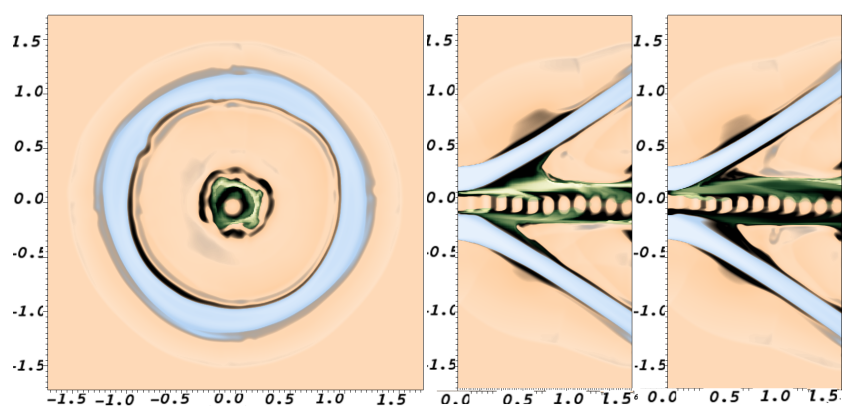

(c) z-y plane, $x=1.5 \times 10^{15} \mathrm{~cm}$ (d) x-y plane, $\mathrm{z}=0 \mathrm{~cm} ; x-z$ plane, $y=0 \mathrm{~cm}$
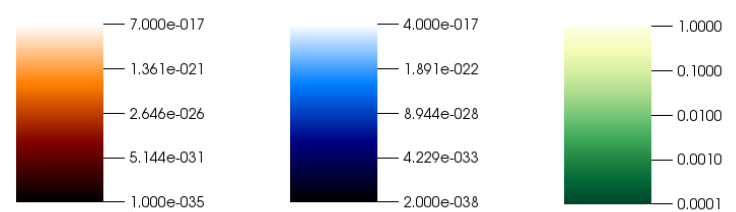

(e) $\log \left(\right.$ density, $\left.\mathrm{gcm}^{-3}\right)$ (f) $\quad \log \left(\mathrm{H}_{2} \quad\right.$ density, $\quad(\mathrm{g}) \log$ (ion fraction) $\left.\mathrm{gcm}^{-3}\right)$

Figure 4. Circumbinary Model s067.3.2: 0.75 AU Binary, atomicmolecular outflow, density cross-sections at simulation time 87.5 years. Axis scales are in units of $10^{15} \mathrm{~cm}$. Underlying density plot is fully opaque. For clarity, $\mathrm{H}_{2}$ and ionisation fraction overplots are at ramped opacity; $100 \%$ opacity at max value, transparent at minimum value.

6 AU followed by a small recovery before another sharp fall to $73 \%$ below inlet value by $11 \mathrm{AU}$.

This explains how the ionised sheath comes to envelop the jet column in the Circumbinary Scenario (including s067.3.3 which has no molecular component). As the centre of the $3.67 \mathrm{AU}$ radius jet inlet describes a $0.75 \mathrm{AU}$ orbit about the barycentre, the periphery of the jet is subject to a continual strong oblique shock, almost as abrupt as the main jet bow shock, in contact with the ambient medium that refocuses the jet inwards. The expansion of the hot gas drives a crossing shock inwards towards the jet centre. Furthermore this shock is a feature that screws around the jet as the motion of the jet carries the disturbance downstream. Figure 8 shows this explicitly for individual fluid elements that we have tracked as they are convected downstream.

Can this model be distinguished from the Co-orbital Scenario? A direct comparison is displayed in Fig 9 where the ion density is displayed as contours. There are three major differences. Firstly, the circumbinary model generates a jet which is less streamlined in axial velocity although after $100 \mathrm{AU}$ the difference in width is not an identifying difference. Secondly, the ion density is much higher within the sheath of the atomic jet, typically by a factor of 100 . This generates observable emission as will be elucidated below. Thirdly, there are weakly ionised arms branching off above and below the jet in the circumbinary model which represents material entrained into the boundary layer of the slow-moving molecular flow. This is mainly due to the proximity of the disc which has an inner edge at 9 AU from the binary barycentre.

\subsection{Longitudinal Analysis of Circumbinary Models}

The jet and molecular outflow do not strongly interact in the Circumbinary Scenario. This contrasts with the co-orbital case of $\mathrm{Pa}$ per 1 where the jet has to penetrate through the molecular cone as it sweeps around. The result is that the analysis of quantities in one-dimensional plots along or transverse to the axis is relatively simple.

The density along the axis is shown in Fig 10 at five times each separated by 24.5 days. The behaviour follows that expected for a pulsed molecular jet (Smith et al. 1997), showing that the pulses expand once the material has been swept up into the compressed knots and the internal shocks weaken. The proper motions of the density peaks remain very close to the average jet speed of $275 \mathrm{~km} \mathrm{~s}^{-1}$

The recollimation shoulder is the most striking new effect of the molecular outflow. As shown in Figure 11 , at a distance $\mathrm{x} \approx$ $55 \mathrm{AU}$, in the presence of the surrounding molecular outflow, the jet column and hot cocoon recollimates, with the cross-sectional area falling off and then stabilising.

At distances $\mathrm{x} \lesssim 55 \mathrm{AU}$, we see from Figure 12 that the ionisation and temperature are lower in the dual outflow circumbinary model than in the single outflow model, but from $55 \mathrm{AU}$ onwards this trend reverses as the ionisation in s067.3.2 dramatically rises. Thus, the molecular outflow confines the ambient medium, maintaining a high pressure which restricts the atomic jet after the initial rapid expansion.

\section{SYNTHETIC IMAGING AND MAPS}

Emission line properties for atomic and molecular species can be derived via post-processing of the physical variables on using statistical weights. For this purpose, we have developed an IDL code , MULTISNTH, to generate images, position-velocity diagrams, channel maps and mass-velocity profiles. In Figure 13, we present images of the $\mathrm{H} \alpha 656 \mathrm{~nm}$ emission superimposed on contour maps of the emission from the $\mathrm{CO} \mathrm{J}=2-1$ rotational transition at $231 \mathrm{GHz}$.

One fascinating feature is the appearance of a wide opening angle to the $\mathrm{H} \alpha$ jet. This occurs out to $\sim 15 \mathrm{AU}$ before abrupt collimation takes place (Panels (c) and (d)). This structure develops from the initial cylindrical jet structure (Panel (a)). The $\mathrm{H} \alpha$ distribution is not completely smooth with some dark patches visible and lateral protruding bright patches. However, when the molecular flow is added, the $\mathrm{H} \alpha$ jet is squeezed in the first $55 \mathrm{AU}$ before widening.

The CO cone develops at a speed of $\sim 10 \mathrm{~km} \mathrm{~s}^{-1}$. The structure remains geometrically similar, consistent with ballistic motion. The outflow remains highly symmetric and aligned with the jet to within $2^{\circ}$ : the motion of the atomic jet has a small but measurable influence on the CO.

The $\mathrm{H} \alpha$ jet does not display the underlying dense knots. This 

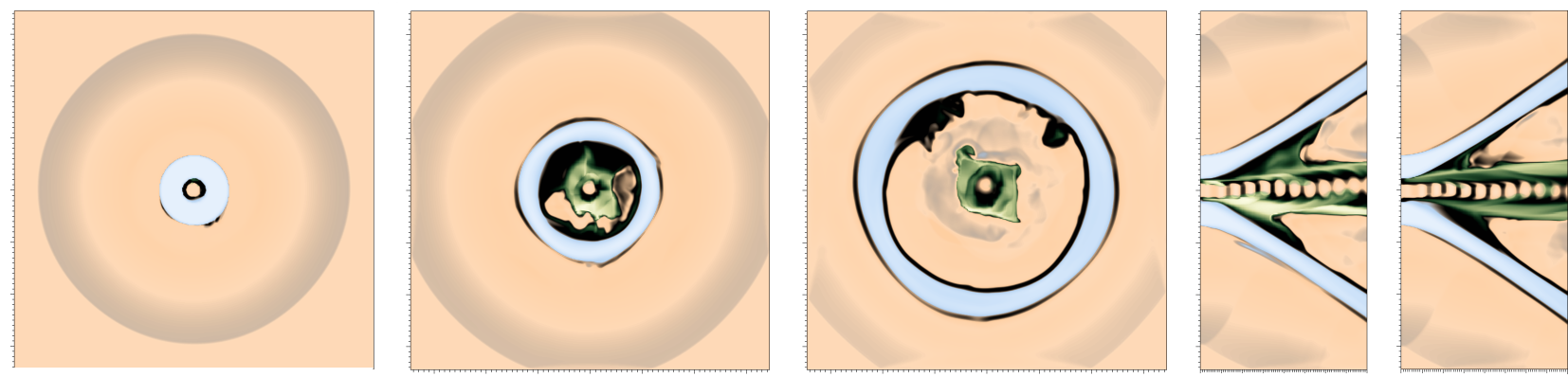

(a) density and composition at $\mathrm{x}=0 \mathrm{~cm}$ (b) density and composition, 50AU

(c) density and composition, 100AU (d) density and composition, xz plane

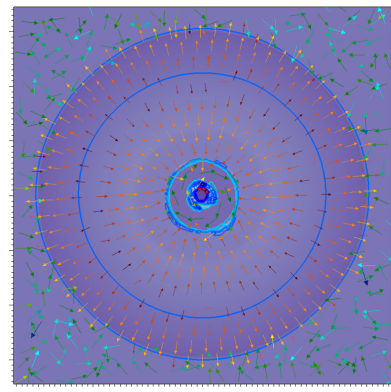

(e) $\mathrm{p}, \mathrm{T}$ and $\mathrm{v}$ at $\mathrm{x}=0 \mathrm{~cm}$

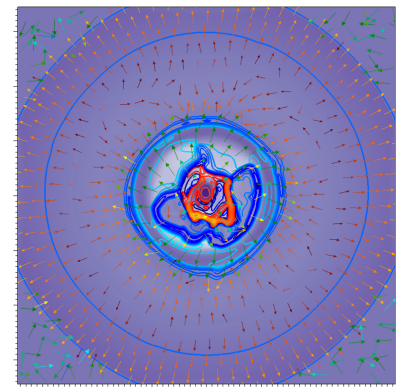

(f) $\mathrm{p}, \mathrm{T}$ and $\mathrm{v}$ at $\mathrm{x}=50 \mathrm{AU}$

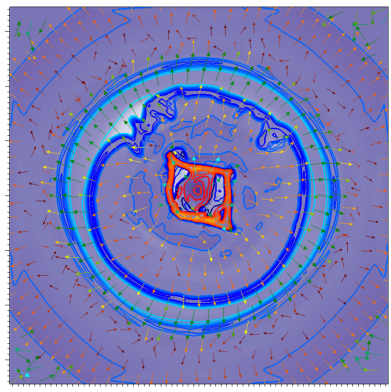

(g) p, T and $\mathrm{v}$ at $\mathrm{x}=100 \mathrm{AU}$
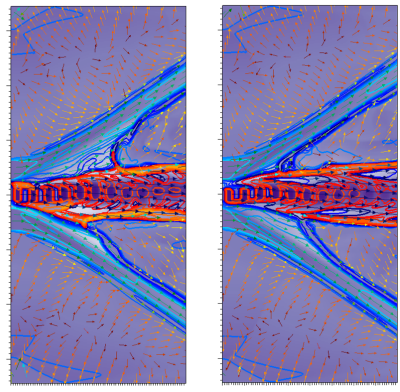

(h) p, T and $v$ in $x y$ and $x z$ planes

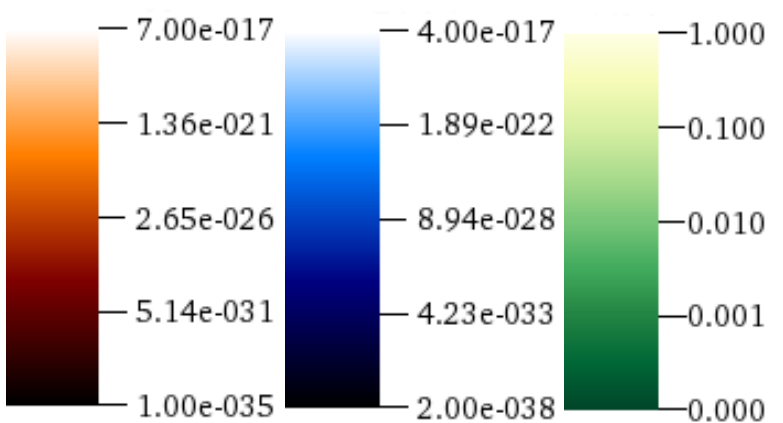

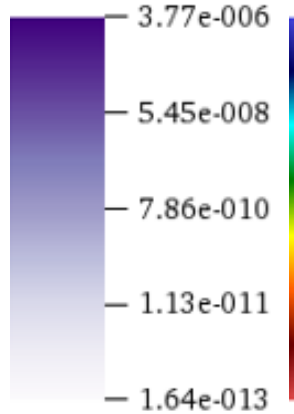

(1) pressure

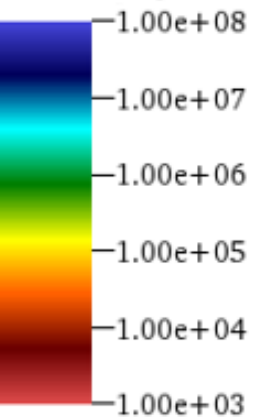

(m) velocity vectors

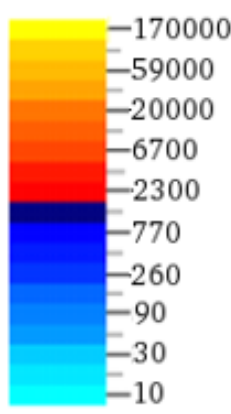

(n) temperature contours

(i) density, $\mathrm{H}$

(j) density, $\mathrm{H}_{2}$

(k) ion fraction

Figure 5. Cross-sections of physical parameters for the Circumbinary Scenario atomic-molecular outflow at simulation time 175 years. Axis scales are in units of $10^{15} \mathrm{~cm}$. Ambient medium is atomic hydrogen with trace molecular hydrogen formed during the simulation. Underlying density plot is fully opaque.

emission is submerged by that caused by the rapid orbit which drives oblique shock waves in the sheath and then into the jet column. This smooth appearance is at odds with the knots detected in HH 30 which suggests that such a rapid orbiting motion of the jet source is not present.

\section{CONCLUSIONS}

The interaction of a jet from one component of a young binary with the heavy outflow from a circumbinary disc has been explored. Several important assumptions were necessary to enable the three dimensional cooling and chemistry to be reasonably resolved. Firstly, the molecular outflow is assumed to be accelerated from a region of the inner disc while the atomic jet is generated from the primary star which orbits on a period of one year.

The outflow axes are assumed to be aligned and the magnetic field is assumed to be dynamically negligible on scales exceeding $10 \mathrm{AU}$. Thus, the hydrodynamic simulations belong to scales exceeding $10 \mathrm{AU}$ whereas the magnetic-field driven launch could be confined to within 0.1 AU. Nevertheless, the field could remain dynamically important on the $10-100 \mathrm{AU}$ scale. The initial ambient medium was taken to be uniform and atomic; the modelled outflows were run for sufficient simulation time to fill the domain. We restrict the model to the inner $107 \mathrm{AU}$ in order to incorporate the wide-angle molecular flow within the limits of available computing resources.

The main result derives from the rapid orbiting nozzzle from which the atomic jet is fired. The jet channel orbits rapidly, deflecting off the ambient medium, creating a sheath of ionised highpressure gas. The jet diverges with oblique shocks creating a warm jet that is significantly wider than the original nozzle. The jet begins to recollimate after expanding to $\sim 15-20 \mathrm{AU}$ at a distance of $\sim 15 \mathrm{AU}$. 


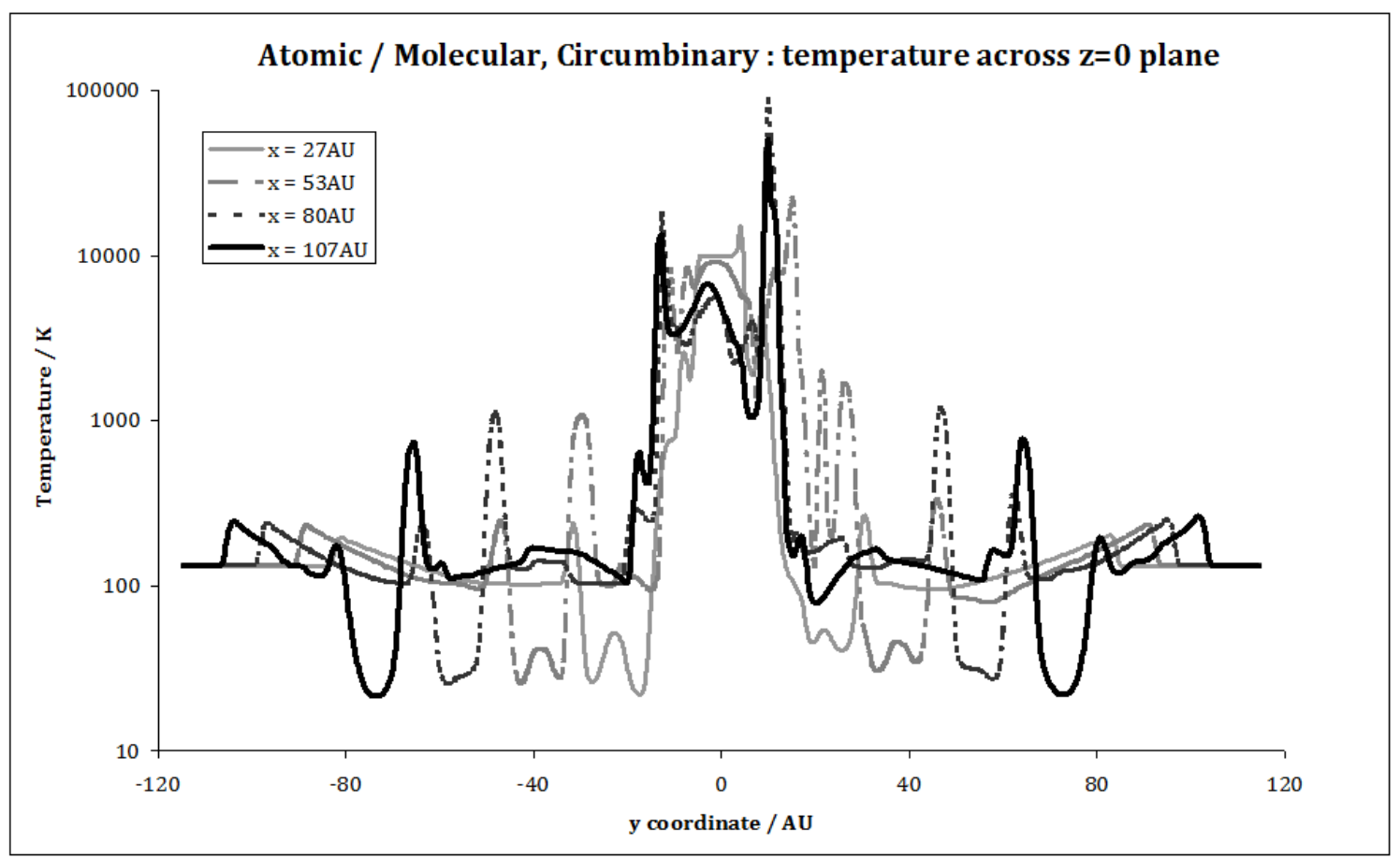

Figure 6. Temperature profiles across the $\mathrm{z}=0$ central plane at selected distances from the inlet (x-axis values) for the circumbinary model s067.3.2.

\section{Velocity Signal, Velocity-Pulsed Atomic Jet: Centre vs Periphery}

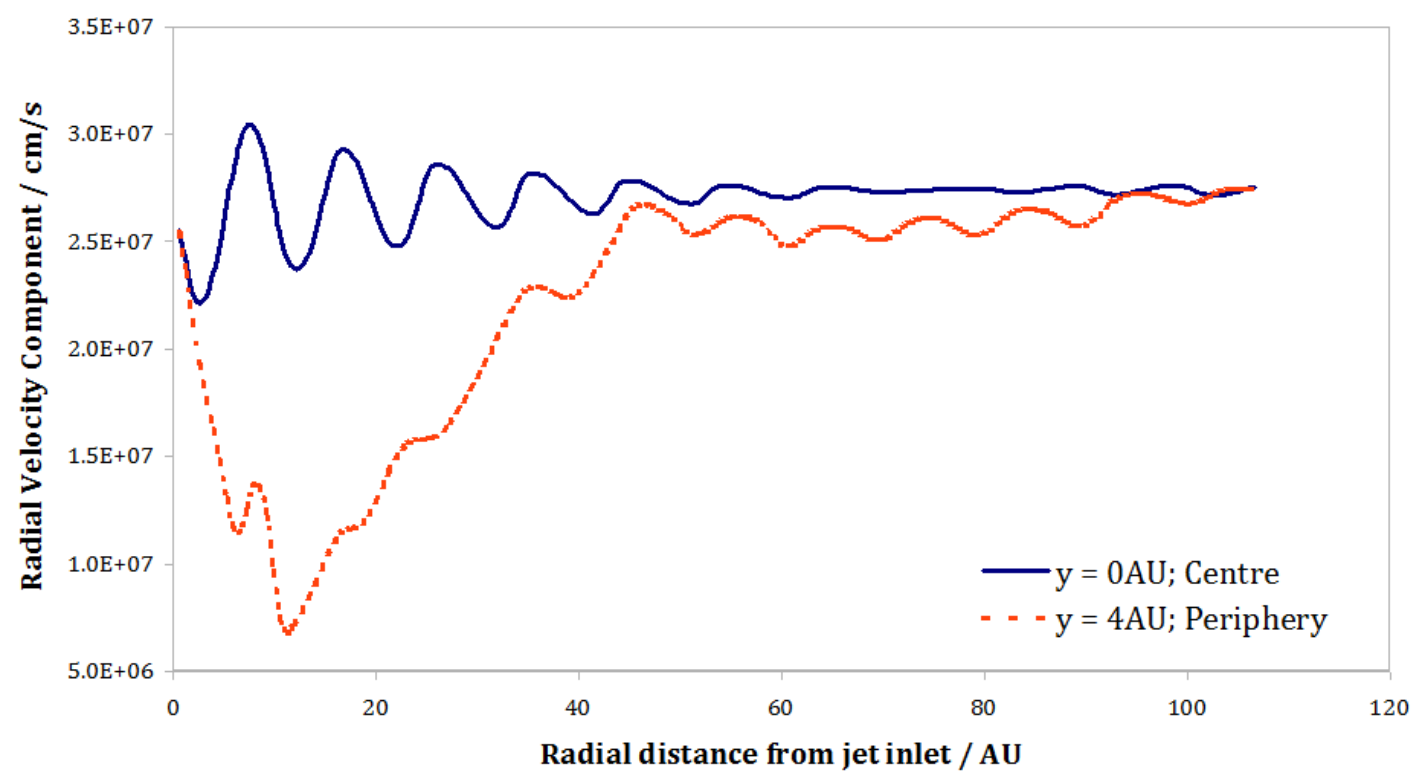

Figure 7. Centre-line vs Peripheral velocity signal along the axis of the pulsed atomic jet in the dual atomic-molecular, circumbinary model s067.3.2. These can be seen to correspond to the emerging ionisation region in the bottom left corner of Fig. 8

In the presence of the molecular outflow, trapped material aids in the confinement of the jet which then recollimates further downstream - at $55 \mathrm{AU}$ under the conditions taken here. In all the conditions, however, the velocity pulses in the jet do not lead to detectable knots in the $\mathrm{H} \alpha$ emission. Instead, the ionisation, caused by the oblique shocks, overwhelms the internal structure in the jet core.

Given these characteristics of the atomic jet, we should exclude the present Circumbinary Scenario as an interpretation of $\mathrm{HH} 30$. To work, we require a binary with a period roughly ten times longer in order for the jet dynamical time to far exceed the 

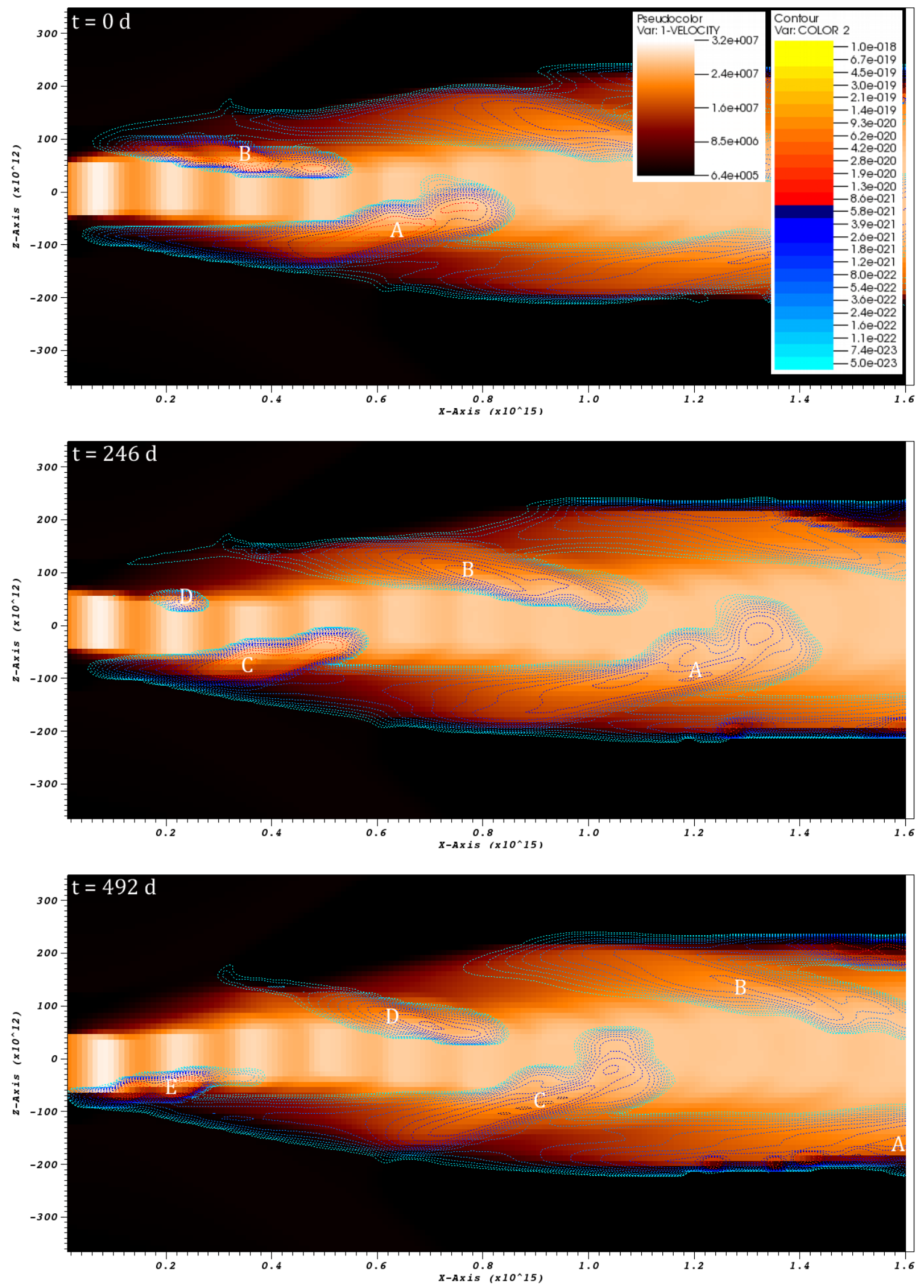

Figure 8. Formation and propagation of ionised regions on the periphery of the atomic jet in the model s067.3.2. Fluid elements A, B, C, D are regions of strong ionisation; in fact cross-sections through a continuous shock that winds around the jet column. Underlying colour plot is the $\mathrm{V}_{x}$ component $\left(\mathrm{cm} \mathrm{s}^{-1}\right)$. Overlaid contours $\left(\mathrm{g} \mathrm{cm}^{-3}\right)$ correspond to partial density of ionised Hydrogen. Cross-sections are at $\mathrm{z}=0$ and axes are $\mathrm{cm}$ scale. 


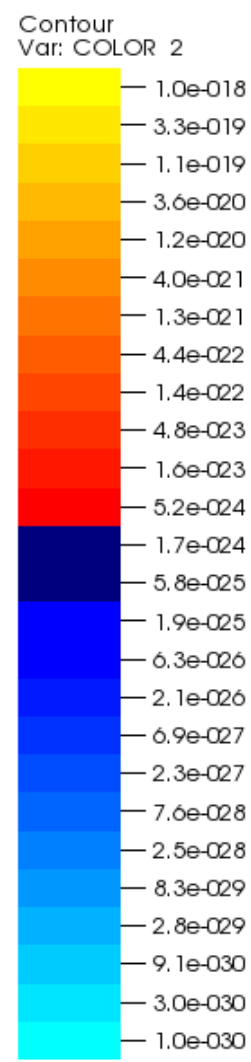

Pseudocolor
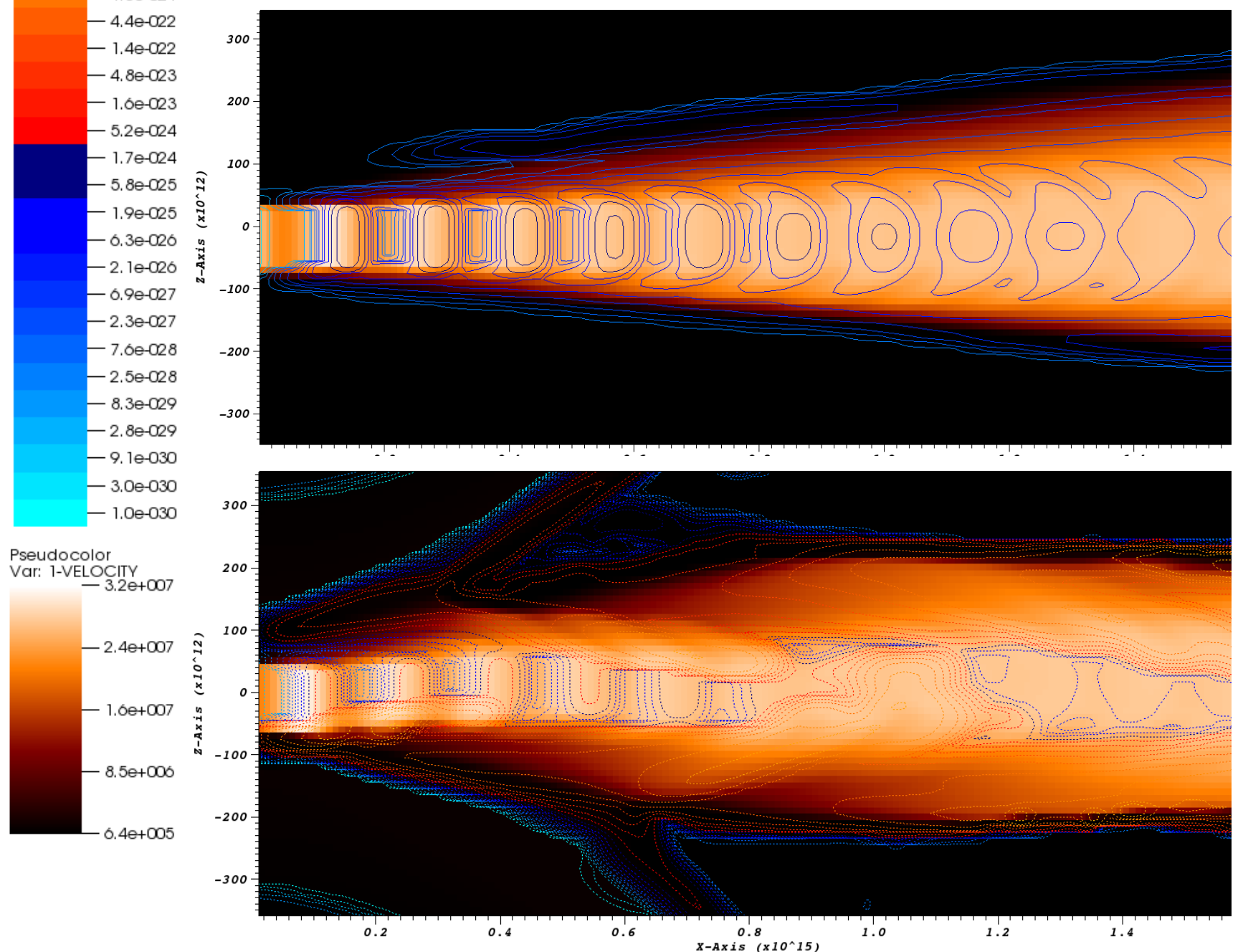

Figure 9. Comparison of ionisation modes in Co-orbital Scenario s066.2.4 (upper panel) vs Circumbinary Scenario s067.3.2 (lower panel). Colour plot is the $\mathrm{V}_{x}$ component $\left(\mathrm{cm} \mathrm{s}^{-1}\right)$; contours $\left(\mathrm{g} \mathrm{cm}^{-3}\right)$ are partial density of ionised Hydrogen; axes are $\mathrm{cm}$ scale. In Fig.8, the contour scale excludes regions of low ionisation to highlight the peripheral screw-thread ionisation shock; here the range is expanded to include lower ion densities. The weakly ionised 'arms' branching off from above and below the jet in the circumbinary model are material entrained into the boundary layer of the slow-moving molecular flow.

orbital time. That implies that the inner edge of the wind-generating circumbinary disc is also situated at a larger distance where the rotation speed is far less. It is unlikely that molecular outflow speeds of order $10 \mathrm{~km} \mathrm{~s}^{-1}$ would then result.

On the other hand, the Co-orbital Scenario faces similar problems, as discussed in Paper 1. In that work, it was shown that the molecular outflow inevitably disrupts the atomic jet.

A resolution would involve a scenario with the present circumbinary molecule flow. The properties of the $\mathrm{CO}$ molecular outflow generated in this Circumbinary Scenario are consistent with the parameter set derived from the latest ALMA data (Louvet et al. 2018). However, to simultaneously avoid the high-excitation atomic sheath and highlight the jet knots, the atomic jet must be generated with less agitation. That requires a low-speed orbiting body which could easily be achieved by reconsideration of the binary parameters. A systematic parameter study is necessary to elucidate this.

Some well-studied jets, including HH 30, show relatively high ionisation and wide opening angles at their base (Ray et al. 1996; Bacciotti et al. 2000; Woitas et al. 2002). To also produce visible knots could be achieved with higher amplitude velocity pulsations combined with pressure pulses within the jet. Or, if the primary star is more massive, then its orbiting path will be reduced, leading to less disruption and a diminished ionised sheath.

The present model may be relevant to interpret the HL Tau jet which displays a high ionised sheath with a low excitation 


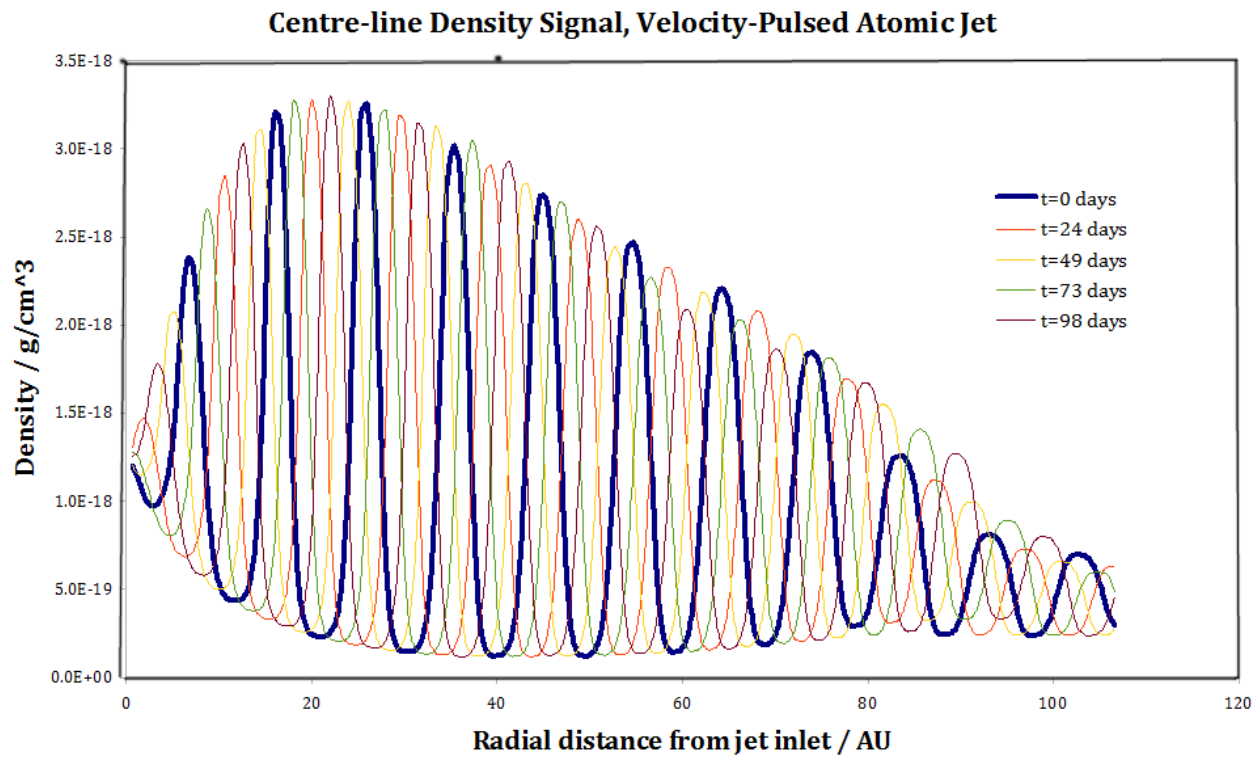

Figure 10. Centre-line density signal along the axis of the pulsed atomic jet in the dual atomic-molecular, Circumbinary Scenario s067.3.2. An additional four time frames are included to show signal propagation.

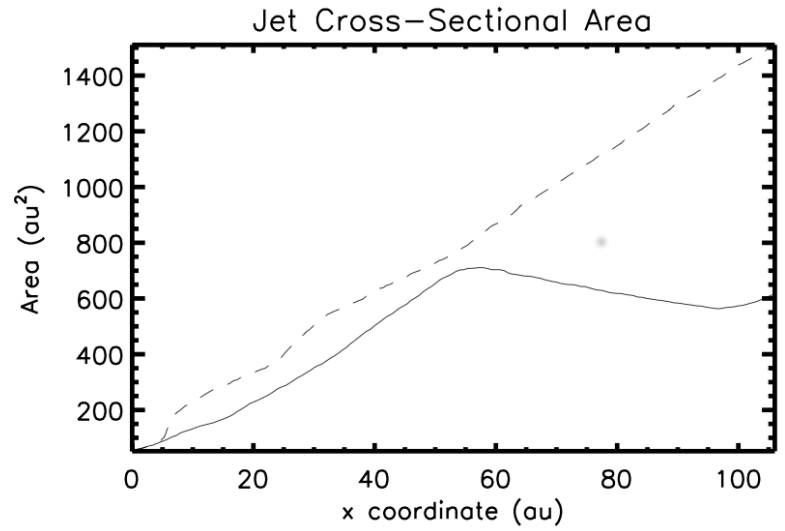

Figure 11. Circumbinary Scenario: atomic jet cross-sectional area and distance along the propagation axis at the time 87.5 years. Model numbers: s067.3.2 (solid line, with molecular outflow) and s067.3.3 (dashed line, without).

spine (Movsessian et al. 2007). This is observed on scales of order 1,000 AU and the triggering outflow may be attributed to an outflow from nearby XZ Tau rather than an HL Tau wind. In addition, it should be noted that there is no evidence that HL Tau is a binary.

The present choice of initial conditions has a strong bearing on the results. It is clear that the high speed and neutral atomic component will lead to enhanced high ionisation regions if the jet is not uniform. Here, the orbital motion of the jet source creates a disturbed sheath which channels energy from the bulk flow into thermal modes. However, the fraction of energy dissipated per unit length and the amount then channelled into ionisation rather than kinetic energy are important factors. The potential degeneracy in the parameter space that this causes will include the orbital period with shorter periods generating narrower sheaths but dissipation over a shorter jet length.

The fact that stars commonly form not only as members of
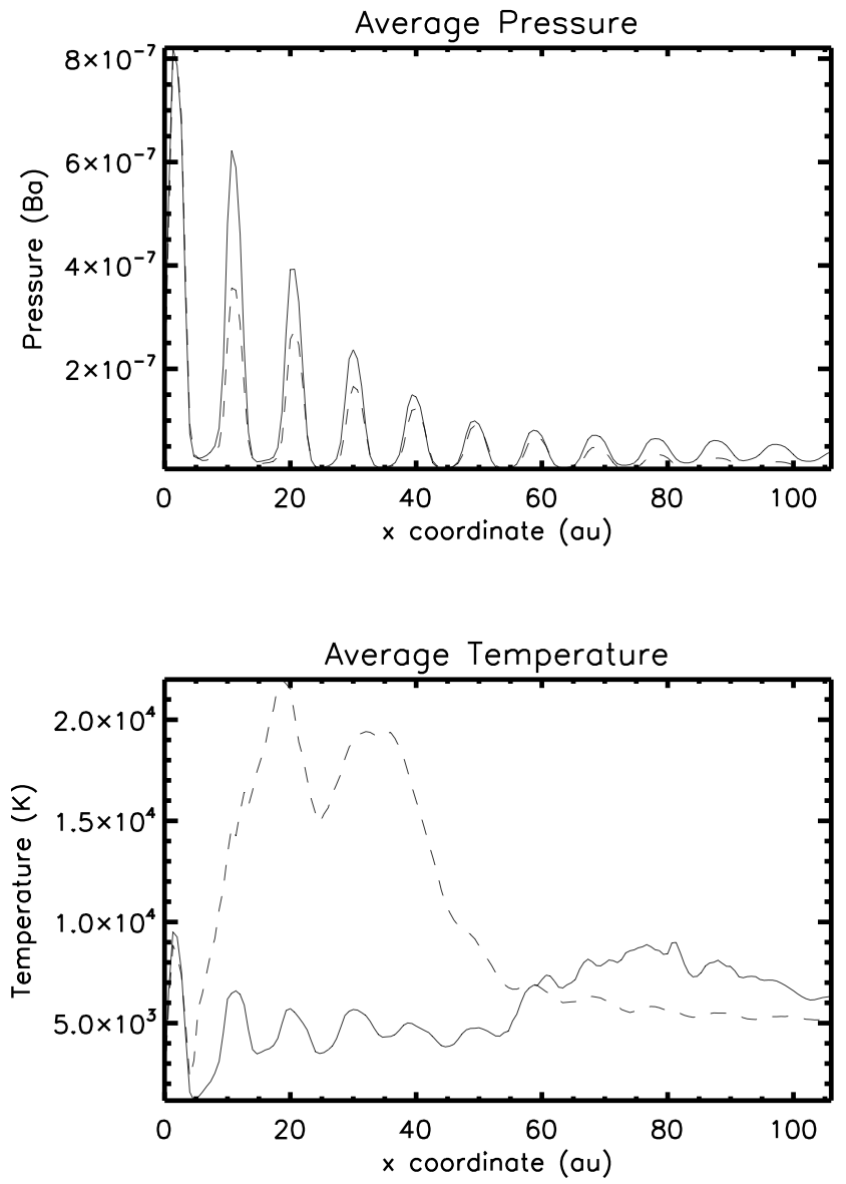

Figure 12. Circumbinary Scenario: atomic jet pressure and temperature along the propagation axis at the time 87.5 years. Model numbers: s067.3.2 (solid line, with molecular outflow) and s067.3.3 (dashed line, without). 

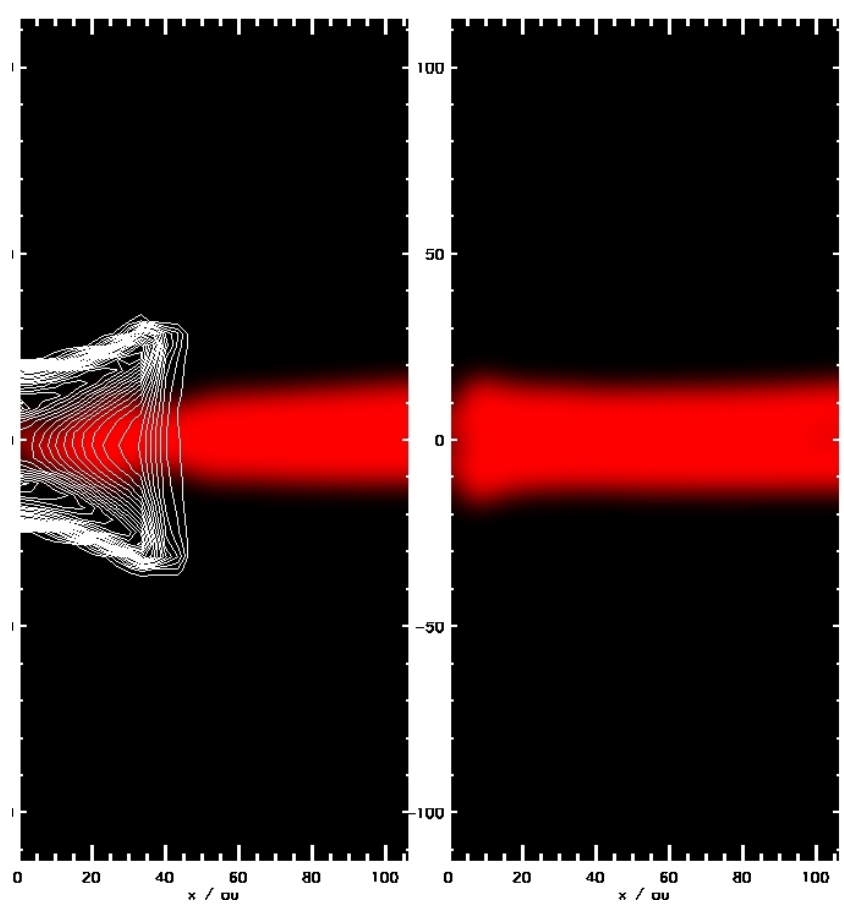

(a) 22 years
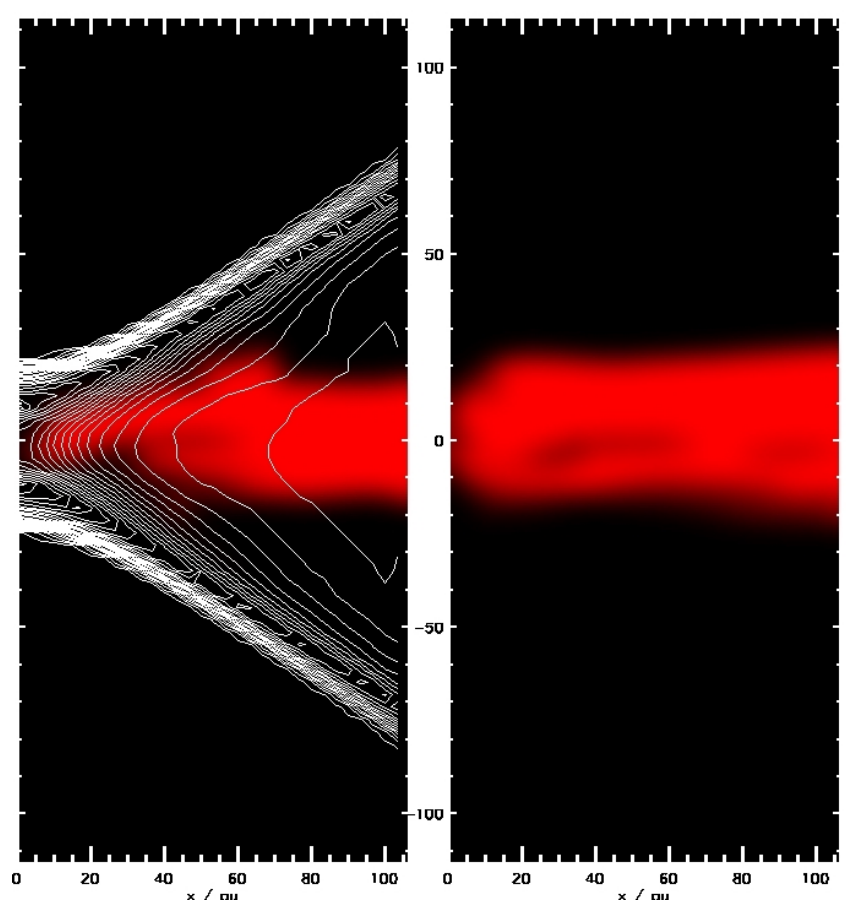

(c) 88 years
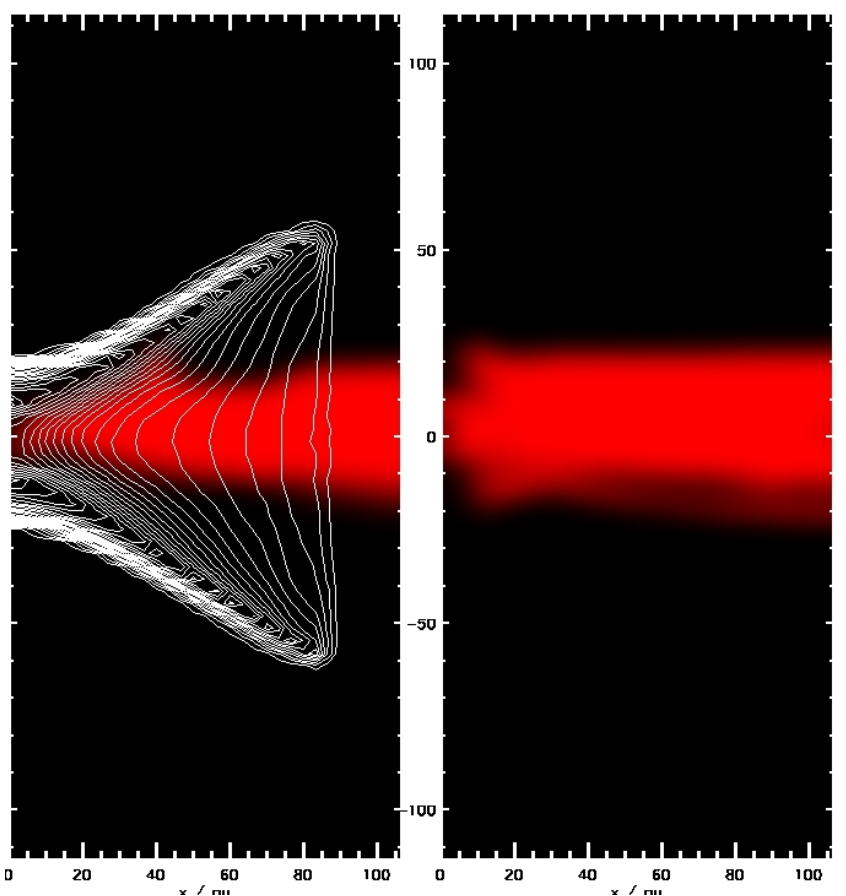

(b) 44 years

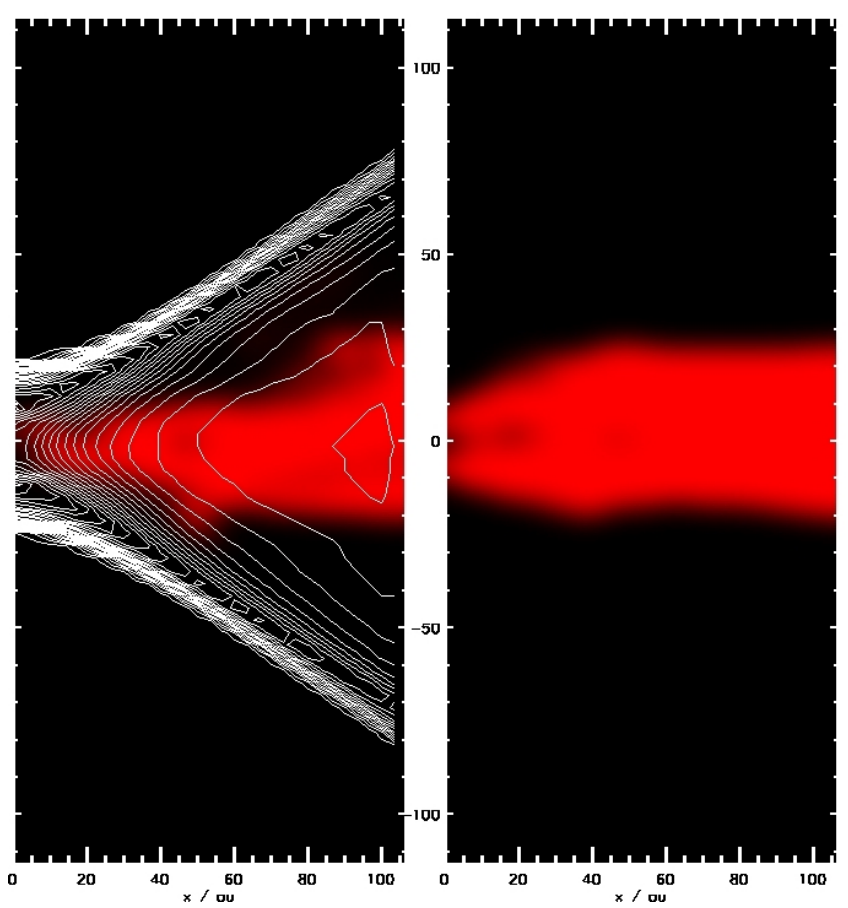

(d) 176 years

Figure 13. Synthetic $\mathrm{H} \alpha$ images and $\mathrm{CO}$ contours at the four indicated times from the Circumbinary Scenario with dual outflows (left sub-panels) and with just the atomic jet (right sub-panels) The optical emission is smoothed with $14 \mathrm{AU}$ radius to match HST/WFPC2 pixel resolution of $0.1^{\prime \prime}$ at $140 \mathrm{pc}$. Contours indicate molecular material (unsmoothed) from the $\mathrm{CO} \mathrm{J}=2-1$ transition.

binaries or of higher order systems but often as close binaries has long been established (Brandner \& Zinnecker 1997). The stars can be at distinct evolutionary stages and can be as close as assumed in these simulations (Hartigan \& Kenyon 2003).

To conclude, the wide molecular flow from the circumbinary disc disturbs the orbiting atomic jet by squeezing the outer sheath of ionised gas. This is seen here to dominate the ionisation structure and the $\mathrm{H} \alpha$ emission making the knots harder to distinguish. The orbiting atomic jet is itself very different from a fixed atomic jet as it scrapes a much wider channel around a fast spine, generating the high excitation sheath. The excitation level within atomic microjets will no doubt be explored in the coming years, led by the James 
Webb Space Telescope, the Giant Magellan Telescope and the European Extremely Large Telescope. These are capable of resolving structure on the scales diagnosed by these simulations.

\section{ACKNOWLEDGEMENTS}

We thank SEPnet and the University of Portsmouth for supplying infrastructure. We also thank Simon Glover for providing support with the code.

\section{REFERENCES}

Anglada G., López R., Estalella R., Masegosa J., Riera A., Raga A. C., 2007, AJ, 133, 2799

Aronow R. A., Herbst W., Hughes A. M., Wilner D. J., Winn J. N., 2018, AJ, 155, 47

Bacciotti F., Mundt R., Ray T. P., Eislöffel J., Solf J., Camezind M., 2000, ApJ, 537, L49

Bate M. R., Tricco T. S., Price D. J., 2014, MNRAS, 437, 77

Blandford R. D., Payne D. G., 1982, MNRAS, 199, 883

Brandner W., Zinnecker H., 1997, A\&A, 321, 220

Estalella R., López R., Anglada G., Gómez G., Riera A., Carrasco-González C., 2012, AJ, 144, 61

Gardner C. L., Jones J. R., Vargas P. B., 2018, Rev. Mex. Astron. Astrofis., 54,317

Glover S. C. O., Mac Low M.-M., 2007, ApJS, 169, 239

Hartigan P., Kenyon S. J., 2003, ApJ, 583, 334

Hartigan P., Morse J., 2007, ApJ, 660, 426

Hayes J. C., Norman M. L., Fiedler R. A., Bordner J. O., Li P. S., Clark S. E., ud-Doula A., Mac Low M.-M., 2006, ApJS, 165, 188

Hirano S., Machida M. N., 2019, MNRAS, 485, 4667

Krist J. E., Stapelfeldt K. R., Hester J. J., Healy K., Dwyer S. J., Gardner C. L., 2008, AJ, 136, 1980

Louvet F., Dougados C., Cabrit S., Mardones D., Ménard F., Tabone B., Pinte C., Dent W. R. F., 2018, A\&A, 618, A120

Lynch C. J. R., Smith M. D., Glover S. C. O., 2019, MNRAS, p. 2590

Machida M. N., 2014, ApJ, 796, L17

Machida M. N., Basu S., 2019, ApJ, 876, 149

Machida M. N., Inutsuka S.-i., Matsumoto T., 2008, ApJ, 676, 1088

McCaughrean M. J., Rayner J. T., Zinnecker H., 1994, ApJ, 436, L189

Movsessian T. A., Magakian T. Y., Bally J., Smith M. D., Moiseev A. V., Dodonov S. N., 2007, A\&A, 470, 605

Murphy G. C., Lery T., O’Sullivan S., Spicer D., Bacciotti F., Rosen A., 2008, A\&A, 478, 453

Pety J., Gueth F., Guilloteau S., Dutrey A., 2006, A\&A, 458, 841

Raga A. C., Canto J., Binette L., Calvet N., 1990, ApJ, 364, 601

Ray T. P., Mundt R., Dyson J. E., Falle S. A. E. G., Raga A. C., 1996, ApJ, 468, L103

Shibata K., Uchida Y., 1985, PASJ, 37, 31

Smith M. D., 2004, "The origin of stars”. Michael D. Smith. London (UK): Imperial College Press

Smith M. D., Suttner G., Zinnecker H., 1997, A\&A, 320, 325

Tambovtseva L. V., Grinin V. P., 2008, MNRAS, 387, 1313

Tomida K., Tomisaka K., Matsumoto T., Hori Y., Okuzumi S., Machida M. N., Saigo K., 2013, ApJ, 763, 6

Woitas J., Eislöffel J., Mundt R., Ray T. P., 2002, ApJ, 564, 834

Zinnecker H., McCaughrean M. J., Rayner J. T., 1998, Nature, 394, 862

de Miera F. C.-S., Kóspál Á., Ábrahám P., Liu H. B., Takami M., 2019, The Astrophysical Journal, 882, L4 
Webb Space Telescope, the Giant Magellan Telescope and the European Extremely Large Telescope. These are capable of resolving structure on the scales diagnosed by these simulations.

\section{ACKNOWLEDGEMENTS}

We thank SEPnet and the University of Portsmouth for supplying infrastructure. We also thank Simon Glover for providing support with the code.

\section{REFERENCES}

Anglada G., López R., Estalella R., Masegosa J., Riera A., Raga A. C., 2007, AJ, 133, 2799

Aronow R. A., Herbst W., Hughes A. M., Wilner D. J., Winn J. N., 2018, AJ, 155, 47

Bacciotti F., Mundt R., Ray T. P., Eislöffel J., Solf J., Camezind M., 2000, ApJ, 537, L49

Bate M. R., Tricco T. S., Price D. J., 2014, MNRAS, 437, 77

Blandford R. D., Payne D. G., 1982, MNRAS, 199, 883

Brandner W., Zinnecker H., 1997, A\&A, 321, 220

Estalella R., López R., Anglada G., Gómez G., Riera A., Carrasco-González C., 2012, AJ, 144, 61

Gardner C. L., Jones J. R., Vargas P. B., 2018, Rev. Mex. Astron. Astrofis., 54,317

Glover S. C. O., Mac Low M.-M., 2007, ApJS, 169, 239

Hartigan P., Kenyon S. J., 2003, ApJ, 583, 334

Hartigan P., Morse J., 2007, ApJ, 660, 426

Hayes J. C., Norman M. L., Fiedler R. A., Bordner J. O., Li P. S., Clark S. E., ud-Doula A., Mac Low M.-M., 2006, ApJS, 165, 188

Hirano S., Machida M. N., 2019, MNRAS, 485, 4667

Krist J. E., Stapelfeldt K. R., Hester J. J., Healy K., Dwyer S. J., Gardner C. L., 2008, AJ, 136, 1980

Louvet F., Dougados C., Cabrit S., Mardones D., Ménard F., Tabone B., Pinte C., Dent W. R. F., 2018, A\&A, 618, A120

Lynch C. J. R., Smith M. D., Glover S. C. O., 2019, MNRAS, p. 2590

Machida M. N., 2014, ApJ, 796, L17

Machida M. N., Basu S., 2019, ApJ, 876, 149

Machida M. N., Inutsuka S.-i., Matsumoto T., 2008, ApJ, 676, 1088

McCaughrean M. J., Rayner J. T., Zinnecker H., 1994, ApJ, 436, L189

Movsessian T. A., Magakian T. Y., Bally J., Smith M. D., Moiseev A. V., Dodonov S. N., 2007, A\&A, 470, 605

Murphy G. C., Lery T., O’Sullivan S., Spicer D., Bacciotti F., Rosen A., 2008, A\&A, 478, 453

Pety J., Gueth F., Guilloteau S., Dutrey A., 2006, A\&A, 458, 841

Raga A. C., Canto J., Binette L., Calvet N., 1990, ApJ, 364, 601

Ray T. P., Mundt R., Dyson J. E., Falle S. A. E. G., Raga A. C., 1996, ApJ, 468, L103

Shibata K., Uchida Y., 1985, PASJ, 37, 31

Smith M. D., 2004, "The origin of stars”. Michael D. Smith. London (UK): Imperial College Press

Smith M. D., Suttner G., Zinnecker H., 1997, A\&A, 320, 325

Tambovtseva L. V., Grinin V. P., 2008, MNRAS, 387, 1313

Tomida K., Tomisaka K., Matsumoto T., Hori Y., Okuzumi S., Machida M. N., Saigo K., 2013, ApJ, 763, 6

Woitas J., Eislöffel J., Mundt R., Ray T. P., 2002, ApJ, 564, 834

Zinnecker H., McCaughrean M. J., Rayner J. T., 1998, Nature, 394, 862

de Miera F. C.-S., Kóspál Á., Ábrahám P., Liu H. B., Takami M., 2019, The Astrophysical Journal, 882, L4 